\title{
Chaos in two black holes with next-to-leading order spin-spin interactions
}

\author{
Guoqing Huang, Xiaoting Ni, Xin $\mathrm{Wu}^{\mathrm{a}}$ \\ Department of Physics, Nanchang University, Nanchang 330031, China
}

Received: 3 March 2014 / Accepted: 29 July 2014 / Published online: 13 August 2014

(c) The Author(s) 2014. This article is published with open access at Springerlink.com

\begin{abstract}
We take into account the dynamics of a complete third post-Newtonian conservative Hamiltonian of two spinning black holes, where the orbital part arrives at the third post-Newtonian precision level and the spin-spin part with the spin-orbit part includes the leading-order and nextto-leading-order contributions. It is shown through numerical simulations that the next-to-leading-order spin-spin couplings play an important role in chaos. A dynamical sensitivity to the variation of single parameter is also investigated in some cases. In particular, there are a number of observable orbits whose initial radii are large enough and which are chaotic before coalescence.
\end{abstract}

\section{Introduction}

Massive binary black-hole systems are likely the most promising sources to be used for future gravitational wave detectors. The successful detection of the waveforms means using matched-filtering techniques to best separate a faint signal from the noise and requires a very precise modeling of the expected waveforms. Post-Newtonian (PN) approximations can satisfy this requirement. Up to now, high-precision PN templates have already been known for the non-spin part up to $3.5 \mathrm{PN}$ order (i.e the order $1 / c^{7}$ in the formal expansion in powers of $1 / c^{2}$ with $c$ being the speed of light) [1,2], the spin-orbit part up to 3.5PN order including the leading-order (LO, 1.5PN), next-to-leading-order (NLO, 2.5PN), and nextto-next-to-leading-order (NNLO, 3.5PN) interactions [3-6], and the spin-spin part up to 4PN order consisting of the LO (2PN), NLO (3PN), and NNLO (4PN) couplings [7-9].

However, an extremely sensitive dependence on initial conditions as the basic feature of chaotic systems would pose a challenge to the implementation of such matched filters, since the number of filters required to detect these waveforms is exponentially large with increasing detection sensitivity.

a e-mail: xwu@ncu.edu.cn
This has led some authors to focus on research of chaos in the orbits of two spinning black holes. Chaos was first found and confirmed in the 2PN Lagrangian approximation of comparable mass binaries with the LO spin-orbit and LO spin-spin effects [10]. Moreover, it was reported in [11] that the presence of chaos should be ruled out in these systems because no positive Lyapunov exponents could be found. As an answer to this claim, $[12,13]$ obtained some positive Lyapunov exponents and pointed out these zero Lyapunov exponents of [11] due to the less rigorous calculation of the Lyapunov exponents of two nearby orbits with unapt rescaling. In fact, the conflicting results on Lyapunov exponents are because the two papers $[11,12]$ used different methods to compute their Lyapunov exponents, as was mentioned in [14]. Schnittman and Rasio [11] computed the stabilizing limit values of Lyapunov exponents, and [12] worked out the slopes of the fit lines. This is the so-called doubt regarding different chaos indicators causing two distinct claims on the chaotic behavior. Besides this, there was a second point of doubt on different dynamical approximations giving the same physical system distinct dynamical behaviors. The 2PN harmoniccoordinates Lagrangian formulation of the two-black-hole system with the LO spin-orbit couplings of one body spinning allows for chaos [15], but the 2PN ADM (ArnowittDeser-Misner) coordinates Hamiltonian does not [16,17]. Levin [18] thought that there is no formal conflict between them since the two approaches are not exactly but approximately equal, and different dynamical behaviors between the two approximately related systems are permitted according to the dynamical system theory. Seen from the canonical, conjugate spin coordinates [19], the former non-integrability and the latter integrability are clearer. As extensions, any PN conservative Hamiltonian binary system with one body spinning and a conservative Hamiltonian of two bodies spinning without the constraint of equal mass (or with the spin-orbit couplings not restricted to the leading order) are still integrable. Recently, [20,21] argued for the integrability of the 
2PN Hamiltonian without the spin-spin couplings and with the NLO and/or NNLO spin-orbit contributions included. On the contrary, the corresponding Lagrangian counterpart with spin effects limited to the spin-orbit interactions up to the NLO terms exhibits the stronger chaoticity [22]. A third point of doubt relates to the different dependence of chaos on a single dynamical parameter or initial condition. The description of the chaotic regions and chaotic parameter spaces in [15] is inconsistent with that in [23]. The different claims are regarded to be correct according to the statement of [24] that chaos does not depend only on a single physical parameter or initial condition but a complicated combination of all parameters and initial conditions.

It is worth emphasizing that the spin-spin effects are the most important source for causing chaos in spinning compact binaries, but they were only restricted to the LO term in the published papers on research of the chaotic behavior. It should be significant to discuss the NLO spin-spin couplings included in the contribution of chaos. For the sake of this, we shall consider a complete 3PN conservative Hamiltonian of two spinning black holes, where the orbital part is up to the 3PN order and the spin-spin part as well as the spin-orbit part includes the LO and NLO interactions. In this way, we want to know whether the inclusion of the NLO spin-spin couplings has an effect on chaos, and whether there is chaos before the coalescence of the binaries.

\section{Third post-Newtonian order Hamiltonian approach}

It is too difficult to strictly describe the dynamics of a system of two mass comparable spinning black holes in general relativity. Instead, the PN approximation method is often used. Suppose that the two bodies have masses $m_{1}$ and $m_{2}$ with $m_{1} \leq m_{2}$. Other mass parameters are the total mass $M=m_{1}+m_{2}$, the reduced mass $\mu=m_{1} m_{2} / M$, the mass ratio $\beta=m_{1} / m_{2}$, and the mass parameter $\eta=$ $\mu / M=\beta /(1+\beta)^{2}$. As to other specified notations, a threedimensional vector $\boldsymbol{r}$ represents the relative position of body 1 to body 2, its unit radial vector is $\boldsymbol{n}=\boldsymbol{r} / \boldsymbol{r}$ with the radius $r=|\boldsymbol{r}|$, and $\boldsymbol{p}$ stands for the momenta of body 1 relative to the center. The momenta, distances, and time $t$ are, respectively, measured in terms of $\mu, M$, and $M$. Additionally, geometric units, $c=G=1$, are adopted. The two spin vectors are $\mathbf{S}_{i}=S_{i} \hat{\mathbf{S}}_{i}(i=1,2)$ with unit vectors $\hat{\mathbf{S}}_{i}$ and the spin magnitudes $S_{i}=\chi_{i} m_{i}^{2} / M^{2}\left(0 \leq \chi_{i} \leq 1\right)$. In ADM coordinates, the system can be expressed as the dimensionless conservative 3PN Hamiltonian,

$$
\begin{aligned}
H\left(\mathbf{r}, \mathbf{p}, \mathbf{S}_{1}, \mathbf{S}_{2}\right)= & H_{\mathrm{o}}(\mathbf{r}, \mathbf{p})+H_{\mathrm{So}}\left(\mathbf{r}, \mathbf{p}, \mathbf{S}_{1}, \mathbf{S}_{2}\right) \\
& +H_{\mathrm{SS}}\left(\mathbf{r}, \mathbf{p}, \mathbf{S}_{1}, \mathbf{S}_{2}\right) .
\end{aligned}
$$

In the following, we write its detailed expressions although they are too long.
For the conservative case, the orbital part $H_{\mathrm{o}}$ does not include the dissipative $2.5 \mathrm{PN}$ term (which is the leadingorder radiation damping level) but the Newtonian term $H_{\mathrm{N}}$ and the PN contributions $H_{1 \mathrm{PN}}, H_{2 \mathrm{PN}}$, and $H_{3 \mathrm{PN}}$, that is,

$H_{\mathrm{O}}=H_{\mathrm{N}}+H_{1 \mathrm{PN}}+H_{2 \mathrm{PN}}+H_{3 \mathrm{PN}}$.

As given in [25], they are

$$
\begin{aligned}
H_{\mathrm{N}}= & \frac{\boldsymbol{p}^{2}}{2}-\frac{1}{r}, \\
H_{1 \mathrm{PN}}= & \frac{1}{8}(3 \eta-1) \boldsymbol{p}^{4}-\frac{1}{2}\left[(3+\eta) \boldsymbol{p}^{2}+\eta(\boldsymbol{n} \cdot \boldsymbol{p})^{2}\right] \frac{1}{r} \\
& +\frac{1}{2 r^{2}}, \\
H_{2 \mathrm{PN}}= & \frac{1}{16}\left(1-5 \eta+5 \eta^{2}\right) \boldsymbol{p}^{6}+\frac{1}{8}\left[\left(5-20 \eta-3 \eta^{2}\right) \boldsymbol{p}^{4}\right. \\
& \left.-2 \eta^{2}(\boldsymbol{n} \cdot \boldsymbol{p})^{2} \boldsymbol{p}^{2}-3 \eta^{2}(\boldsymbol{n} \cdot \boldsymbol{p})^{4}\right] \frac{1}{r}+\frac{1}{2}\left[(5+8 \eta) \boldsymbol{p}^{2}\right. \\
& \left.+3 \eta(\boldsymbol{n} \cdot \boldsymbol{p})^{2}\right] \frac{1}{r^{2}}-\frac{1}{4}(1+3 \eta) \frac{1}{r^{3}},
\end{aligned}
$$

$$
\begin{aligned}
H_{3 \mathrm{PN}}= & \frac{1}{128}\left(-5+35 \eta-70 \eta^{2}+35 \eta^{3}\right) \boldsymbol{p}^{8}+\frac{1}{16}[(-7 \\
& \left.+42 \eta-53 \eta^{2}-5 \eta^{3}\right) \boldsymbol{p}^{6}+(2-3 \eta) \eta^{2}(\boldsymbol{n} \cdot \boldsymbol{p})^{2} \\
& \left.\times \boldsymbol{p}^{4}+3(1-\eta) \eta^{2}(\boldsymbol{n} \cdot \boldsymbol{p})^{4} \boldsymbol{p}^{2}-5 \eta^{3}(\boldsymbol{n} \cdot \boldsymbol{p})^{6}\right] \frac{1}{r} \\
& +\left[\frac{1}{16}\left(-27+136 \eta+109 \eta^{2}\right) \boldsymbol{p}^{4}+\frac{1}{16}(17\right. \\
& \left.+30 \eta) \eta(\boldsymbol{n} \cdot \boldsymbol{p})^{2} \boldsymbol{p}^{2}+\frac{1}{12}(5+43 \eta) \eta(\boldsymbol{n} \cdot \boldsymbol{p})^{4}\right] \frac{1}{r^{2}} \\
& +\left\{\left[-\frac{25}{8}+\left(\frac{1}{64} \pi^{2}-\frac{335}{48}\right) \eta-\frac{23}{8} \eta^{2}\right] \boldsymbol{p}^{2}\right. \\
& \left.+\left(-\frac{85}{16}-\frac{3}{64} \pi^{2}-\frac{7}{4} \eta\right) \eta(\boldsymbol{n} \cdot \boldsymbol{p})^{2}\right\} \frac{1}{r^{3}} \\
& +\left[\frac{1}{8}+\left(\frac{109}{12}-\frac{21}{32} \pi^{2}\right) \eta\right] \frac{1}{r^{4}} .
\end{aligned}
$$

The spin-orbit part $H_{\text {so }}$ is a linear function of the two spins. It is the sum of the LO spin-orbit term $H_{\text {so }}^{\mathrm{LO}}$ and the NLO

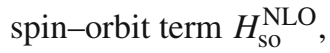

$H_{\mathrm{so}}=H_{\mathrm{so}}^{\mathrm{LO}}\left(\mathbf{r}, \mathbf{p}, \mathbf{S}_{1}, \mathbf{S}_{2}\right)+H_{\mathrm{so}}^{\mathrm{NLO}}\left(\mathbf{r}, \mathbf{p}, \mathbf{S}_{1}, \mathbf{S}_{2}\right)$.

Nagar [6] gave their expressions:

$H_{\mathrm{So}}=\frac{1}{r^{3}}\left[g(\boldsymbol{r}, \boldsymbol{p}) \boldsymbol{S}+g^{*}(\boldsymbol{r}, \boldsymbol{p}) \boldsymbol{S}^{*}\right] \cdot \boldsymbol{L}$,

where the related notations are

$S=S_{1}+S_{2}, S^{*}=\frac{1}{\beta} S_{1}+\beta S_{2}$,

$g(\boldsymbol{r}, \boldsymbol{p})=2+\left[\frac{19}{8} \eta \mathbf{p}^{2}+\frac{3}{2} \eta(\mathbf{n} \cdot \mathbf{p})^{2}-(6+2 \eta) \frac{1}{r}\right]$, 


$$
\begin{aligned}
g^{*}(\mathbf{r}, \mathbf{p})= & \frac{3}{2}+\left[-\left(\frac{5}{8}+2 \eta\right) \mathbf{p}^{2}+\frac{3}{4} \eta(\mathbf{n} \cdot \mathbf{p})^{2}\right. \\
& \left.-(5+2 \eta) \frac{1}{r}\right],
\end{aligned}
$$

and the Newtonian-looking orbital angular momentum vector is

$$
\mathbf{L}=\mathbf{r} \times \mathbf{p} .
$$

The constant terms in $g$ and $g^{*}$ correspond to the LO part, and the others to the NLO part.

Similarly, the spin-spin Hamiltonian $H_{\mathrm{sS}}$ also consists of the LO spin-spin coupling term $H_{\mathrm{ss}}^{\mathrm{LO}}$ and the NLO spin-spin coupling term $H_{\mathrm{ss}}^{\mathrm{NLO}}$,

$$
H_{\mathrm{sS}}=H_{\mathrm{sS}}^{\mathrm{LO}}\left(\mathbf{r}, \mathbf{S}_{1}, \mathbf{S}_{2}\right)+H_{\mathrm{sS}}^{\mathrm{NLO}}\left(\mathbf{r}, \mathbf{p}, \mathbf{S}_{1}, \mathbf{S}_{2}\right) \text {. }
$$

The first sub-Hamiltonian reads [25]

$$
H_{\mathrm{sS}}^{\mathrm{LO}}=\frac{1}{2 r^{3}}\left[3\left(\mathbf{S}_{0} \cdot \boldsymbol{n}\right)^{2}-\mathbf{S}_{0}^{2}\right]
$$

with $\mathbf{S}_{0}=\mathbf{S}+\mathbf{S}^{*}$. The second sub-Hamiltonian is made of three parts,

$$
H_{\mathrm{sS}}^{\mathrm{NLO}}=H_{s_{1}^{2} p^{2}}+H_{s_{2}^{2} p^{2}}+H_{s_{1} s_{2} p^{2}} .
$$

They are written in $[7,8]$ as

$$
\begin{aligned}
H_{S_{1}^{2} p^{2}}= & \frac{1}{r^{3}}\left\{\frac { 1 } { 4 \beta ^ { 2 } } \left[\left(\boldsymbol{p}_{1} \cdot S_{1}\right)^{2}+\frac{3}{2}\left(\boldsymbol{p}_{1} \cdot \boldsymbol{n}\right)^{2} S_{1}^{2}\right.\right. \\
& \left.-\frac{3}{2} \boldsymbol{p}_{1}^{2}\left(\boldsymbol{S}_{1} \cdot \boldsymbol{n}\right)^{2}-3\left(\boldsymbol{p}_{1} \cdot \boldsymbol{n}\right)\left(\boldsymbol{S}_{1} \cdot \boldsymbol{n}\right)\left(\boldsymbol{p}_{1} \cdot \boldsymbol{S}_{1}\right)\right] \\
& -\frac{3}{4} \boldsymbol{p}_{2}^{2}\left[\boldsymbol{S}_{1}^{2}-3\left(\boldsymbol{S}_{1} \cdot \boldsymbol{n}\right)^{2}\right] \\
& +\frac{3}{4 \beta}\left[\left(\boldsymbol{p}_{1} \cdot \boldsymbol{p}_{2}\right) \boldsymbol{S}_{\mathbf{1}}^{2}-3\left(\boldsymbol{p}_{1} \cdot \boldsymbol{p}_{2}\right)\left(\boldsymbol{S}_{1} \cdot \boldsymbol{n}\right)^{2}\right. \\
& -2\left(\boldsymbol{p}_{1} \cdot \boldsymbol{n}\right)\left(\boldsymbol{p}_{2} \cdot \boldsymbol{S}_{1}\right)\left(\boldsymbol{S}_{1} \cdot \boldsymbol{n}\right) \\
& +4\left(\boldsymbol{p}_{2} \cdot \boldsymbol{n}\right)\left(\boldsymbol{p}_{1} \cdot \boldsymbol{S}_{1}\right)\left(\boldsymbol{S}_{1} \cdot \boldsymbol{n}\right) \\
& +\left(\boldsymbol{p}_{1} \cdot \boldsymbol{n}\right)\left(\boldsymbol{p}_{2} \cdot \boldsymbol{n}\right) \boldsymbol{S}_{1}^{2} \\
& \left.\left.-5\left(\boldsymbol{p}_{1} \cdot \boldsymbol{n}\right)\left(\boldsymbol{p}_{2} \cdot \boldsymbol{n}\right)\left(\boldsymbol{S}_{1} \cdot \boldsymbol{n}\right)^{2}\right]\right\} \\
H_{S_{2}^{2} p^{2}=} & H_{S_{1}^{2} p^{2}}(1 \leftrightarrow 2), \\
H_{S_{1} S_{2} p^{2}=} & \frac{1}{2 r^{3}}\left\{\frac{3}{2}\left[\left(\boldsymbol{p}_{1} \times \boldsymbol{S}_{1}\right) \cdot \boldsymbol{n}\right]\left[\left(\boldsymbol{p}_{2} \times \boldsymbol{S}_{2}\right) \cdot \boldsymbol{n}\right]\right. \\
& +6\left[\left(\boldsymbol{p}_{2} \times \boldsymbol{S}_{1} \cdot \boldsymbol{n}\right]\left[\left(\boldsymbol{p}_{1} \times \boldsymbol{S}_{2}\right) \cdot \boldsymbol{n}\right]\right. \\
& -15\left(\boldsymbol{S}_{1} \cdot \boldsymbol{n}\right)\left(\boldsymbol{S}_{2} \cdot \boldsymbol{n}\right)\left(\boldsymbol{p}_{1} \cdot \boldsymbol{n}\right)\left(\boldsymbol{p}_{2} \cdot \boldsymbol{n}\right) \\
& -3\left(\boldsymbol{S}_{1} \cdot \boldsymbol{n}\right)\left(\boldsymbol{S}_{2} \cdot \boldsymbol{n}\right)\left(\boldsymbol{p}_{1} \cdot \boldsymbol{p}_{2}\right) \\
& +3\left(\boldsymbol{S}_{1} \cdot \boldsymbol{p}_{2}\right)\left(\boldsymbol{S}_{2} \cdot \boldsymbol{n}\right)\left(\boldsymbol{p}_{1} \cdot \boldsymbol{n}\right) \\
& +3\left(\boldsymbol{S}_{2} \cdot \boldsymbol{p}_{1}\right)\left(\boldsymbol{S}_{1} \cdot \boldsymbol{n}\right)\left(\boldsymbol{p}_{2} \cdot \boldsymbol{n}\right) \\
& +3\left(\boldsymbol{S}_{1} \cdot \boldsymbol{p}_{1}\right)\left(\boldsymbol{S}_{2} \cdot \boldsymbol{n}\right)\left(\boldsymbol{p}_{2} \cdot \boldsymbol{n}\right) \\
& +3\left(\boldsymbol{S}_{2} \cdot \boldsymbol{p}_{2}\right)\left(\boldsymbol{S}_{1} \cdot \boldsymbol{n}\right)\left(\boldsymbol{p}_{1} \cdot \boldsymbol{n}\right) \\
&
\end{aligned}
$$

$$
\begin{aligned}
& -\frac{1}{2}\left(\boldsymbol{S}_{1} \cdot \boldsymbol{p}_{2}\right)\left(\boldsymbol{S}_{2} \cdot \boldsymbol{p}_{1}\right)+\left(\boldsymbol{S}_{1} \cdot \boldsymbol{p}_{1}\right)\left(\boldsymbol{S}_{2} \cdot \boldsymbol{p}_{2}\right) \\
& -3\left(\boldsymbol{S}_{1} \cdot \boldsymbol{S}_{2}\right)\left(\boldsymbol{p}_{1} \cdot \boldsymbol{n}\right)\left(\boldsymbol{p}_{2} \cdot \boldsymbol{n}\right) \\
& \left.+\frac{1}{2}\left(\boldsymbol{S}_{1} \cdot \boldsymbol{S}_{2}\right)\left(\boldsymbol{p}_{1} \cdot \boldsymbol{p}_{2}\right)\right\} \\
& +\frac{3}{2 \beta r^{3}}\left\{-\left[\left(\boldsymbol{p}_{1} \times \boldsymbol{S}_{1}\right) \cdot \boldsymbol{n}\right]\left[\left(\boldsymbol{p}_{1} \times \boldsymbol{S}_{2}\right) \cdot \boldsymbol{n}\right]\right. \\
& +\left(\boldsymbol{S}_{1} \cdot \boldsymbol{S}_{2}\right)\left(\boldsymbol{p}_{1} \cdot \boldsymbol{n}\right)^{2} \\
& \left.-\left(\boldsymbol{S}_{1} \cdot \boldsymbol{n}\right)\left(\boldsymbol{S}_{2} \cdot \boldsymbol{p}_{1}\right)\left(\boldsymbol{p}_{1} \cdot \boldsymbol{n}\right)\right\} \\
& +\frac{3 \beta}{2 r^{3}}\left\{-\left[\left(\boldsymbol{p}_{2} \times \boldsymbol{S}_{2}\right) \cdot \boldsymbol{n}\right]\left[\left(\boldsymbol{p}_{2} \times \boldsymbol{S}_{1}\right) \cdot \boldsymbol{n}\right]\right. \\
& +\left(\boldsymbol{S}_{1} \cdot \boldsymbol{S}_{2}\right)\left(\boldsymbol{p}_{2} \cdot \boldsymbol{n}\right)^{2} \\
& \left.-\left(\boldsymbol{S}_{2} \cdot \boldsymbol{n}\right)\left(\boldsymbol{S}_{1} \cdot \boldsymbol{p}_{2}\right)\left(\boldsymbol{p}_{2} \cdot \boldsymbol{n}\right)\right\} \\
& +\frac{6 M^{2}}{m_{1} m_{2} r^{4}}\left[\left(\boldsymbol{S}_{1} \cdot \boldsymbol{S}_{2}\right)-2\left(\boldsymbol{S}_{1} \cdot \boldsymbol{n}\right)\left(\boldsymbol{S}_{2} \cdot \boldsymbol{n}\right)\right] .
\end{aligned}
$$

Note that $\mathbf{p}_{1}=-\mathbf{p}_{2}=\mathbf{p}$. In particular, the mass factors in Eqs. (13) and (15) are different from those in [7,8] due to the present rescaling actions on the variables, which are $\mathbf{p}_{1} \rightarrow \mu \mathbf{p}_{1}, \mathbf{p}_{2} \rightarrow \mu \mathbf{p}_{2}, r \rightarrow M r$, and $H_{\mathrm{ss}}^{\mathrm{NLO}} \rightarrow \mu H_{\mathrm{ss}}^{\mathrm{NLO}}$. Naturally, $1 / M^{4}$ is a rescaling factor of the action on the spinsquared interactions. This shows that the rescaling factor of the spin variables in the Hamiltonian (1) is $1 / M^{2}$ rather than $1 /(\mu M)$. To put it another way, we should use $\mathbf{S}_{i} \rightarrow M^{2} \mathbf{S}_{i}$ as rescaled spin transformations. In this sense, the conservative system (1) at the 3PN order is no longer dependent on any mass but the mass ratio.

The evolutions of position $\mathbf{r}$ and momentum $\mathbf{p}$ satisfy the canonical equations of the Hamiltonian (1):

$\frac{\mathrm{d} \mathbf{r}}{\mathrm{d} t}=\frac{\partial H}{\partial \mathbf{p}}, \quad \frac{\mathrm{d} \mathbf{p}}{\mathrm{d} t}=-\frac{\partial H}{\partial \mathbf{r}}$.

The spin variables vary with time according to the following relations:

$\frac{\mathrm{d} \mathbf{S}_{i}}{\mathrm{~d} t}=\frac{\partial H}{\partial \mathbf{S}_{i}} \times \mathbf{S}_{i}$.

Besides the two spin magnitudes, there are four conserved quantities in the Hamiltonian (1), including the total energy $E=H$ and three components of the total angular momentum vector $\boldsymbol{J}=\boldsymbol{L}+\boldsymbol{S}$. A fifth constant of motion is absent, so the Hamiltonian (1) is non-integrable. ${ }^{1}$ Its high nonlinearity may imply that it is a richer source for chaos. Next, we shall search for chaos, and particularly investigate the effect of the NLO spin-spin interactions on the dynamics of the system.

\footnotetext{
${ }^{1}$ Based on the idea of [19], the Hamiltonian (1) can be expressed as a completely canonical Hamiltonian with a ten-dimensional phase space when the canonical, conjugate spin coordinates are used instead of the original spin variables. If the system is integrable, at least five independent integrals of motion beyond the constant spin magnitudes are necessary.
} 


\section{Detection of chaos before coalescence}

With numerical simulations, we use some chaos indicators to describe dynamical differences between the NLO spin-spin couplings excluded and included. The appropriate ones of the indicators are selected to study the dependence of chaos on a single parameter when the NLO spin-spin couplings are included so that a lot of chaotic orbits can be conveniently found, and we can further find some of the chaotic orbits before coalescence by estimating the Lyapunov and in-spiral decay times.

\subsection{Comparisons}

Numerical methods are convenient to study the nonlinear dynamics of the Hamiltonian (1). Symplectic integrators are efficient numerical tools since they have good geometric and physical properties, such as the symplectic structure being conserved and energy errors being without secular changes. However, they cannot provide high enough accuracies, and the computations are expensive when the mixed symplectic integration algorithms [21,26] with a composite of the second-order explicit leapfrog symplectic integrator and the second-order implicit midpoint rule are chosen. In this sense, we would prefer to adopt an eighth-(ninth-)order Runge-Kutta-Fehlberg algorithm of variable time steps. In fact, it gives such a high accuracy to the energy error in the magnitude of about order $10^{-13}-10^{-12}$ when the integration time reaches $10^{6}$, as shown in Fig. 1. Here, for orbit 1 we consider that it has initial conditions $(\boldsymbol{p}(0) ; \boldsymbol{r}(0))=$ $(0,0.39,0 ; 8.55,0,0)$, which correspond to the initial eccentricity $e_{0}=0.30$ and the initial semi-major axis $a_{0}=12.2$. Other parameters and initial spin angles are, respectively, $\beta=0.79, \chi_{1}=\chi_{2}=\chi=1.0, \theta_{i}=78.46^{\circ}$, and $\phi_{i}=60^{\circ}$, where the polar angles $\theta_{i}$ and azimuthal angles $\phi_{i}$ satisfy the relations $\hat{\mathbf{S}}_{i}=\left(\cos \phi_{i} \sin \theta_{i}, \sin \phi_{i} \sin \theta_{i}, \cos \theta_{i}\right)$, as commonly used in physics. The NLO spin-spin couplings are not included in Fig. 1a, but in Fig. 1b. It can be seen clearly that the inclusion of the NLO spin-spin cou- plings with a rather long expression decreases only slightly the numerical accuracy. Therefore, our numerical results are shown to be reliable although the energy errors have secular changes.

We apply several chaos indicators to compare dynamical behaviors of orbit 1 according to the two cases without and with the NLO spin-spin couplings. The method of the Poincaré surface of a section can provide a clear description of the structure of phase space of a conservative system whose phase space has four dimensions. However, it is not suitable for such a higher dimensional system (1). Fortunately, power spectra, Lyapunov exponents, and fast Lyapunov indicators would work well in finding chaos regardless of the dimensionality of phase space.

\subsubsection{Power spectrum analysis}

Power spectrum analysis reveals a distribution of various frequencies $\omega$ of a signal $x(t)$. It is the Fourier transformation

$X(\omega)=\int_{-\infty}^{+\infty} x(t) \mathrm{e}^{-i \omega t} \mathrm{~d} t$,

where $i$ is the imaginary unit. In general, the power spectra $X(\omega)$ are discrete for periodic and quasi-periodic orbits but continuous for chaotic orbits. That is to say, the classification of orbits can be distinguished in terms of different features of the spectra. On the basis of this, we know through Fig. 2 that the orbit seems to be regular when the NLO spinspin couplings are not included, but chaotic when the NLO spin-spin couplings are included. Notice that the method of power spectra is only a rough estimation of the regularity and chaoticity of orbits. More reliable chaos indicators are strongly desired.

\subsubsection{Lyapunov exponents}

The maximum Lyapunov exponent is used to measure the average separation rate of two neighboring orbits in the phase space and gives quantitative analysis to the strength of chaos.
Fig. 1 Energy errors of orbit 1 . The NLO spin-spin couplings are not included in $\mathbf{a}$ but in $\mathbf{b}$ (a)

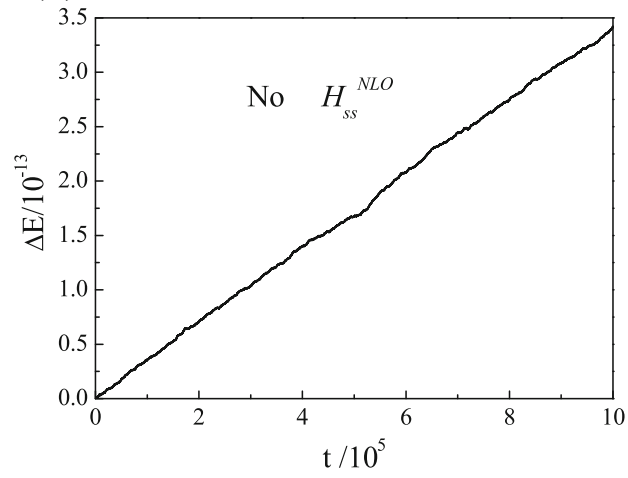

(b)

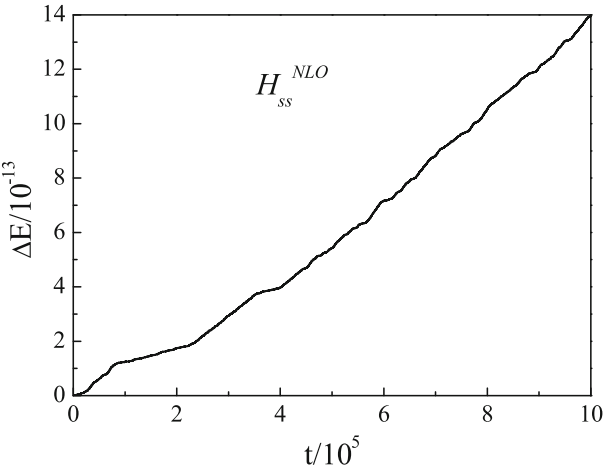


Fig. 2 Power spectra corresponding to Fig. 1

Fig. 3 The maximum Lyapunov exponents $\lambda$ corresponding to Fig. 1 (a)

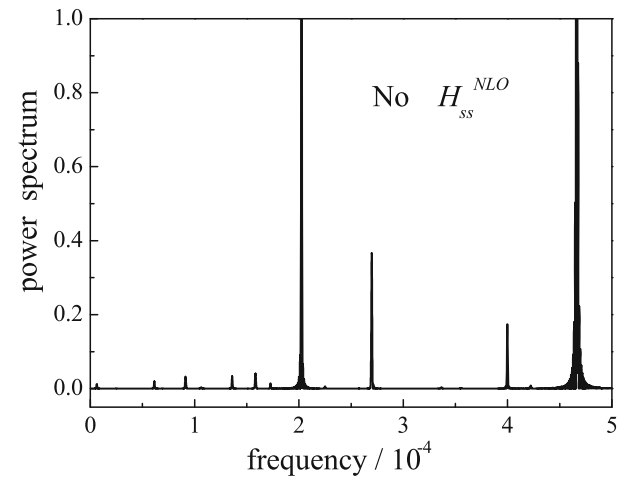

(a)

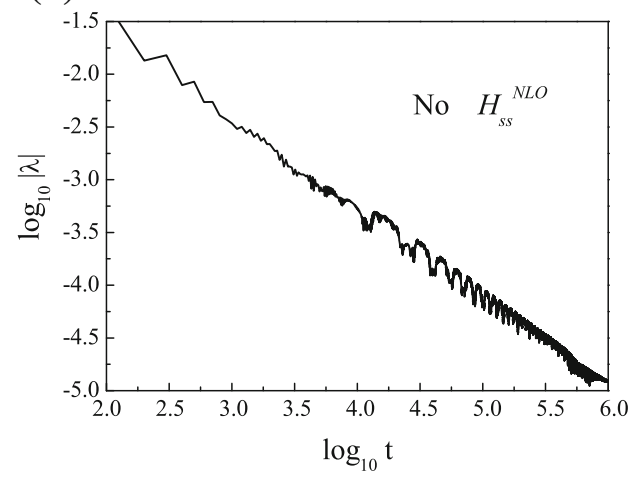

(b)

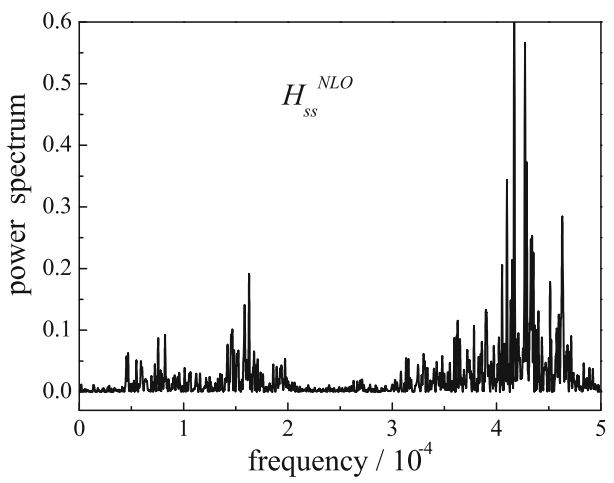

(b)

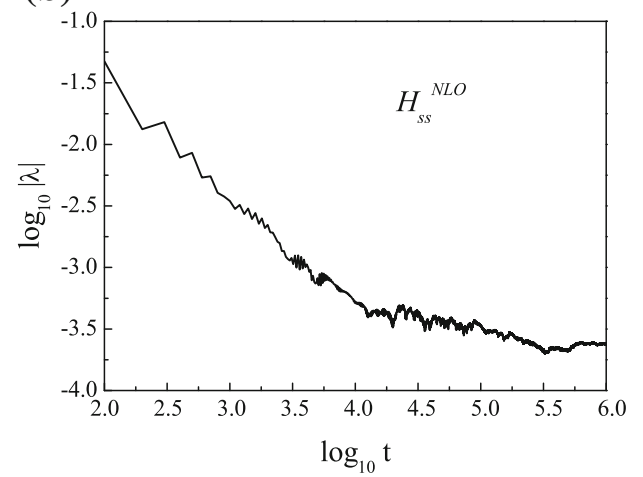

Its calculations are usually based on the variational method and the two-particle method [27]. The former needs solving the variational equations as well as the equations of motion, and the latter needs solving the equations of motion only. Considering the difficulty in deriving the variational equations of a complicated dynamical system, we pay attention to the application of the latter method. In the configuration space, it is defined in [28] as

$\lambda=\lim _{t \rightarrow \infty} \frac{1}{t} \ln \frac{|\Delta \mathbf{r}(t)|}{|\Delta \mathbf{r}(0)|}$,

where $|\Delta \mathbf{r}(0)|$ and $|\Delta \mathbf{r}(t)|$ are the separations between the two neighboring orbits at times 0 and $t$, respectively. The initial distance cannot be too big or too small, and $10^{-8}$ is regarded as to a suitable choice in the double precision [27]. For the sake of the overflow avoided, renormalizations from time to time are vital in the tangent space. A bounded orbit is chaotic if its Lyapunov exponent is positive, but regular when its Lyapunov exponent tends to zero. In this way, we can know from Fig. 3 that orbit 1 is regular for the case without the NLO spin-spin couplings, but chaotic for the case with the NLO spin-spin couplings. Of course, it takes much computational cost to distinguish between the ordered and chaotic cases.

\subsubsection{Fast Lyapunov indicators}

A quicker method to find chaos than the method of Lyapunov exponents is a fast Lyapunov indicator (FLI). This indicator, originally considered to measure the expansion rate of a tangential vector [29], does not need any renormalization, while its modified version dealing with the use of the two-particle method [30] does. The modified version is of the form

FLI $(\mathrm{t})=\log _{10} \frac{|\Delta \mathbf{r}(t)|}{|\Delta \mathbf{r}(0)|}$.

Its computation is based on the following expression:

$\mathrm{FLI}=-k\left(1+\log _{10}|\Delta \mathbf{r}(0)|\right)+\log _{10} \frac{|\Delta \mathbf{r}(t)|}{|\Delta \mathbf{r}(0)|}$,

where $k$ denotes the sequential number of renormalization. The FLI of Fig. 4a, corresponding to Fig. 3a, increases algebraically with logarithmic time $\log _{10} t$, and that of Fig. $4 \mathrm{~b}$, corresponding to Fig. 3b, increases exponentially with logarithmic time. The former indicates the character of order, but the latter the feature of chaos. Only when the integration time adds up to $1 \times 10^{5}$ can the ordered and chaotic behaviors be identified clearly for the use of FLI unlike the application of Lyapunov exponent. There is a threshold value of the FLIs between order and chaos, 5. Orbits whose FLI are larger than 5 are chaotic, whereas those whose FLIs are $<5$ are regular. 
Fig. 4 The fast Lyapunov indicators (FLIs) corresponding to Fig. 1 (a)

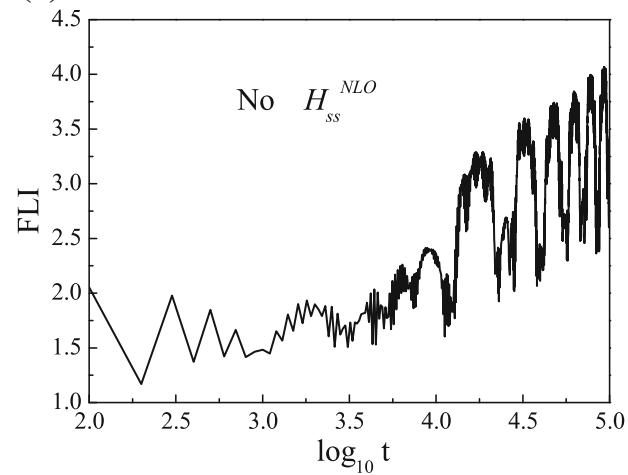

(b)

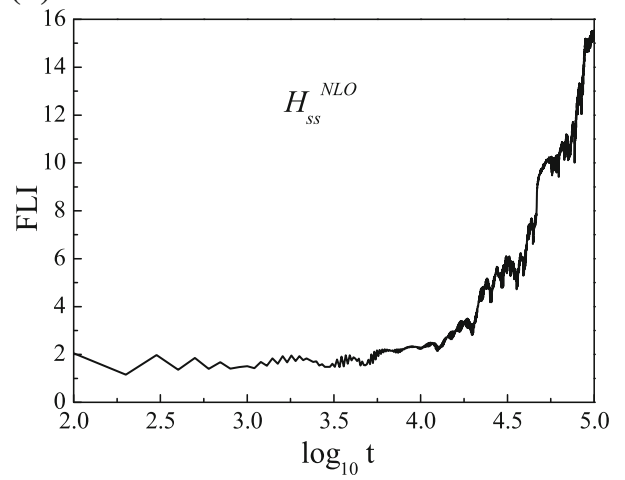

Table 1 Values of FLIs and $\lambda T_{d}$ for different orbits. FLIa corresponds to the NLO spin-spin couplings turned off. FLIb, $\lambda, \lambda d$, and $\lambda T_{d}$ correspond to the NLO spin-spin couplings included

\begin{tabular}{|c|c|c|c|c|c|c|c|c|c|c|c|}
\hline Orbit & $\beta$ & $\chi$ & $x$ & $p_{y}$ & $e_{0}$ & $a_{0}$ & FLIa & FLIb & $\lambda$ & $\lambda_{d}$ & $\lambda T_{d}$ \\
\hline 2 & 0.80 & 1.0 & 8.55 & 0.39 & 0.30 & 12.2 & 4.2 & 14.5 & $3.2 \mathrm{E}-4$ & $8.0 \mathrm{E}-4$ & 0.4 \\
\hline 3 & 0.50 & 0.84 & 14.5 & 0.24 & 0.16 & 12.4 & 3.9 & 21.6 & 4.7E-4 & $5.2 \mathrm{E}-4$ & 0.9 \\
\hline 4 & 0.50 & 0.85 & 14.5 & 0.24 & 0.16 & 12.4 & 4.5 & 15.3 & $3.2 \mathrm{E}-4$ & $5.2 \mathrm{E}-4$ & 0.6 \\
\hline 5 & 0.71 & 0.95 & 17.5 & 0.20 & 0.30 & 13.5 & 3.9 & 17.7 & $3.8 \mathrm{E}-4$ & $5.3 \mathrm{E}-4$ & 0.7 \\
\hline 6 & 0.65 & 0.90 & 35.4 & 0.10 & 0.65 & 21.5 & 4.2 & 9.6 & $1.8 \mathrm{E}-4$ & $3.8 \mathrm{E}-4$ & 0.5 \\
\hline 7 & 0.50 & 0.97 & 18.7 & 0.19 & 0.32 & 14.1 & 9.4 & 24.2 & $5.7 \mathrm{E}-4$ & $4.3 \mathrm{E}-4$ & 1.3 \\
\hline
\end{tabular}

The above numerical comparisons seem to tell us that chaos becomes easier when the NLO spin-spin terms are included. This sounds reasonable. As claimed in [20,21], the system (1) is integrable and not at all chaotic when the spin-spin couplings are turned off. The occurrence of chaos is completely due to the spin-spin couplings, which include particularly the NLO spin-spin contributions, leading to a sharp increase in the strength of nonlinearity. In fact, we employ FLIs to find that there are other orbits (such as orbits 2-6 in Table 1), which are not chaotic for the absence of the NLO spin-spin couplings but for the presence of the NLO spin-spin couplings. In addition, the strength of the chaoticity of orbit 7 increases. To illustrate, the other initial conditions beyond Table 1 are those of orbit 1; the starting spin unit vectors of orbit 2 are those of orbit 1, and those of orbits 3-7 are $\theta_{1}=84.26^{\circ}, \phi_{1}=60^{\circ}, \theta_{2}=84.26^{\circ}$, and $\phi_{2}=45^{\circ}$. Hereafter, only the dynamics of the complete Hamiltonian (1) with the NLO spin-spin effects included is focused on.

\subsection{Lyapunov and in-spiral decay times}

Now we take $\beta=0.5$, the initial conditions $(\boldsymbol{p}(0) ; \boldsymbol{r}(0))=$ $(0,0.19,0 ; 18.7,0,0)$ with the initial eccentricity $e_{0}=0.32$ and the initial semi-major axis $a_{0}=14.1$, and the initial unit spin vectors of orbit 3 . Then we start with the spin parameter $\chi$ at the value 0.2 , which is increased in increments of 0.01 up to a final value of 1 . Finally, dependence of FLI on $\chi$ is plotted in Fig. 5a. This makes it clear that chaos occurs when $\chi \geq 0.45$. Precisely speaking, the larger the spin magnitudes get, the stronger the chaos gets. Note that this dependence of chaos on $\chi$ relies typically on the choice of the initial conditions, the initial unit spin vectors, and the mass ratio parameter. As claimed in [24], there is a different dependence of chaos on $\chi$ if the choice changes. That means that there is not a general result as regards the relationship between chaos and the parameter. On the other hand, taking the initial spin angles of orbit 3 , fixing the spin parameter $\chi=0.95$ and the initial conditions $(\boldsymbol{p}(0) ; \boldsymbol{r}(0))=(0,0.2,0 ; 17.5,0,0)$, which correspond to the initial eccentricity $e_{0}=0.30$ and the initial semi-major axis $a_{0}=13.5$, we study the range of the mass ratio $\beta$ beginning at 0.5 and ending at 1 in increments of 0.01 . At once, the dependence of FLI on $\beta$ can be described in Fig. 5b. There is chaos for most values of $\beta \leq 0.8$ and chaos seems easier for a smaller mass ratio. When the mass ratio is further decreased, all orbits become unstable and tend to mergers, so that whether chaos gets stronger is unclear. Of course, this result is given only under the present initial conditions, initial unit spin vectors, and other parameters, as is described in panel a. Although there is no a universal rule about the dependence of chaos on single parameter or initial condition for any case, insights into the dependence of the dynamics on these parameters are convenient and helpful to search for a large number of chaotic orbits in the case of the given conditions. In this way, we shall further determine that some of them are indeed chaotic before the merger of the binaries. 
Fig. 5 The FLIs as a function of $\chi$ or $\beta$ when the NLO spin-spin interactions are included. All FLIs larger than 5 mean chaos

Fig. 6 The maximum Lyapunov exponents $\lambda$ corresponding to Fig. 5. Note that $\lambda>\lambda_{c}$ means chaos, and $\lambda>\lambda_{d}$ with $\lambda_{d}=1 / T_{d}$ indicates the occurrence of chaos before coalescence of the binaries (a)

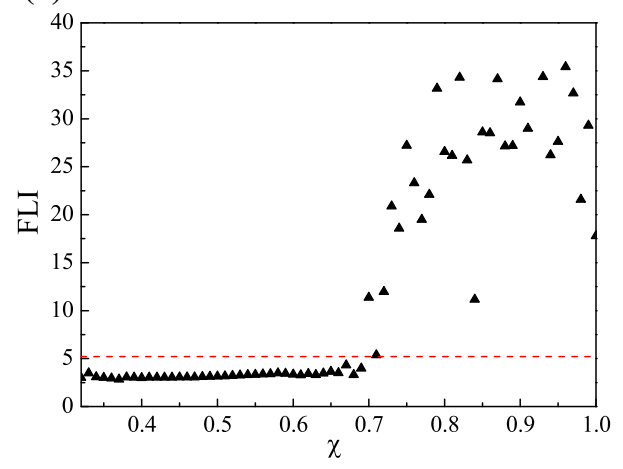

(a)

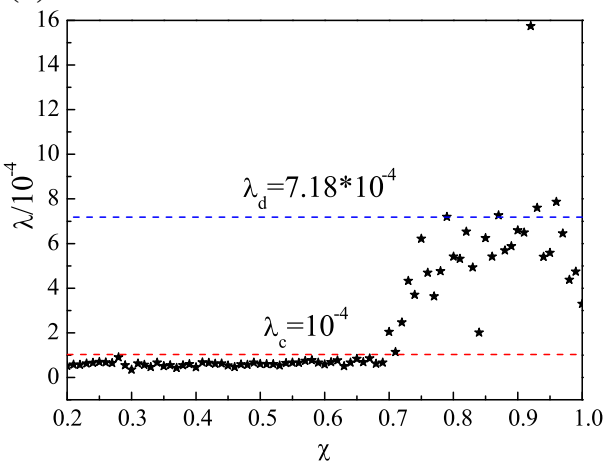

(b)

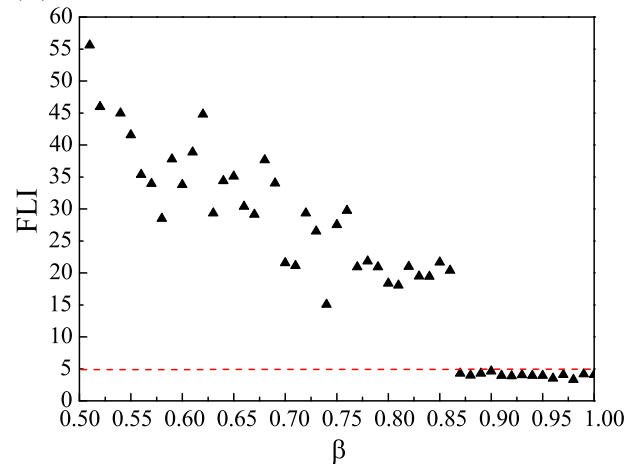

(b)

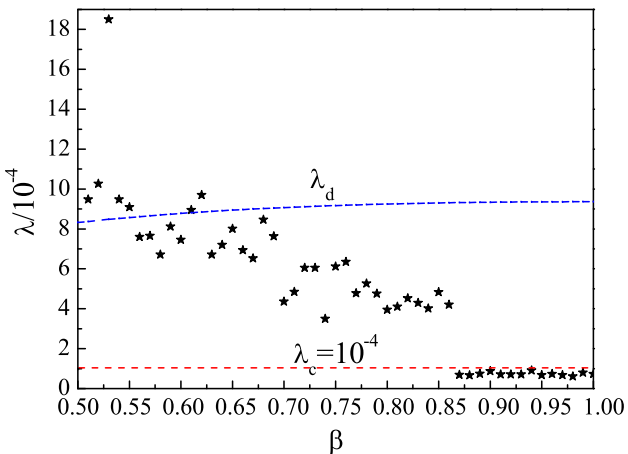

Table 2 Values of $\lambda T_{d}$ for chaotic orbits with big initial radii when the NLO spin-spin contributions are included

\begin{tabular}{llllllllll}
\hline Orbit & $\chi$ & $\beta$ & $x$ & $p_{y}$ & $e_{0}$ & $a_{0}$ & $\lambda$ & $\lambda_{d}$ & $\lambda T_{d}$ \\
\hline 8 & 0.95 & 0.50 & 14.5 & 0.24 & 0.16 & 12.5 & $5.7 \mathrm{E}-4$ & $5.2 \mathrm{E}-4$ & 1.1 \\
9 & 0.94 & 0.50 & 25.5 & 0.14 & 0.50 & 17.0 & $5.7 \mathrm{E}-4$ & $3.8 \mathrm{E}-4$ & 1.5 \\
10 & 0.96 & 0.50 & 25.5 & 0.14 & 0.50 & 17.0 & $4.0 \mathrm{E}-4$ & $3.8 \mathrm{E}-4$ & 1.1 \\
11 & 0.97 & 0.50 & 25.5 & 0.14 & 0.50 & 17.0 & $5.3 \mathrm{E}-4$ & $3.8 \mathrm{E}-4$ & 1.4 \\
12 & 0.90 & 0.50 & 35.4 & 0.10 & 0.65 & 21.5 & $5.2 \mathrm{E}-4$ & $3.6 \mathrm{E}-4$ & 1.4 \\
13 & 0.90 & 0.54 & 35.4 & 0.10 & 0.65 & 21.5 & $6.0 \mathrm{E}-4$ & $3.7 \mathrm{E}-4$ & 1.6 \\
\hline
\end{tabular}

To find the chaotic orbits needed, we have to compare the Lyapunov time $T_{\lambda}=1 / \lambda$ (i.e. the inverse of the Lyapunov exponent) with the in-spiral decay time $T_{d}$, estimated in [31] by

$T_{d}=\frac{12}{19} \frac{c_{0}^{4}}{\gamma} \int_{0}^{e_{0}} \frac{e^{29 / 19}\left[1+(121 / 304) e^{2}\right]^{1181 / 2299}}{\left(1-e^{2}\right)^{3 / 2}} \mathrm{~d} e$,

where the two parameters are

$c_{0}=a_{0}\left(1-e_{0}^{2}\right) e_{0}^{-12 / 19}\left(1+\frac{121}{304} e_{0}^{2}\right)^{-870 / 2299}$

and $\gamma=64 m_{1} m_{2} /\left(5 M^{2}\right)$. When $T_{\lambda}$ is less than $T_{d}$ (or $\lambda T_{d}$ $>1$ ), chaos would be observed. Because $T_{\lambda}=3.0 \times 10^{3}$ and $T_{d}=1.3 \times 10^{3}$ for orbit 1 , the chaoticity cannot be seen before the merger. Values of $\lambda T_{d}$ for orbits 2-7 are listed in Table 1. Clearly, only chaotic orbit 7 is what we expect. Besides these, we plot two panels a and b of Fig. 6 regarding the dependence of the Lyapunov exponent on a single parameter, which correspond, respectively, to Fig. 5a, b. There are two facts to mention. First, the results in Fig. 6 are almost the same as those in Fig. 5. Second, lots of chaotic orbits whose Lyapunov times are many times greater than the in-spiral times should be ruled out, and there are only a small quantity of desired chaotic orbits left.

In order to make the accuracy of the PN approach better, we should choose orbits whose initial radii are larger enough than roughly $10 \mathrm{M}$. Besides the above-mentioned chaotic orbits with initial radii larger than $10 M$ and Lyapunov times less than the in-spiral times, all chaotic orbits in Table 2 are expected. Notice that the other initial conditions of these orbits beyond this table are $y=z=p_{x}=p_{z}=0$, and the starting spin angles are still the same as those of orbit 3. Although an orbit has a large initial radius, it may still be 
chaotic when its initial eccentricity is high enough. This supports the result of [23] that only highly eccentric orbits with very low pericenters could be chaotic. The results about the dependence of chaos on the initial eccentricity in the present paper and the work [23] are really linked to the choice of the other fixed parameters and initial conditions. As claimed in $[15,24]$, the eccentricity alone is not at all responsible for chaos and a general result for the dependence of chaos on the initial eccentricity is absent under various circumstances. Although chaos does not necessarily need a large eccentricity or a small periastron, we still consider the choice of a high eccentricity for our purpose, which is not to obtain a general result as regards the relationship between the initial eccentricity and chaos for any case but to find some chaotic orbits with larger initial radii before the merger. To be compared with our intuition, a high eccentricity seems to make chaos easy in our considered conditions even if the initial radius is large enough. This is because it can get the orbit's periastron close enough in a highly nonlinear regime so that the spinspin coupling contributions become larger and chaos may get stronger.

\section{Conclusions}

This paper is devoted to the study of the dynamics of the complete 3PN conservative Hamiltonian of spinning compact binaries in which the orbital part is accurate to the 3PN order and the spin-spin part as well as the spin-orbit part includes the LO and NLO contributions. Because of the high nonlinearity, the NLO spin-spin couplings included give rise to the occurrence of strong chaos in contrast with those excluded. By scanning a single parameter with the FLIs, we have obtained the dependence of chaos on the parameter for given conditions. It is shown sufficiently that chaos appears more easily for larger spins or smaller mass ratios under the present considered initial conditions, starting unit spin vectors, and other parameters. By means of such a case-by-case scan on chaos versus single parameter, a large number of chaotic orbits can be provided conveniently. Some of them, which become chaotic before the merger, are further given. It is worth noting that an orbit with a large initial radius is still possibly chaotic if its initial eccentricity is high enough. Above all, there are some observable chaotic orbits whose initial radii are suitably large and whose Lyapunov times are less than the corresponding in-spiral times.
Acknowledgments The authors are grateful to a referee for useful suggestions. This research is supported by the Natural Science Foundation of China under Grant Nos. 11173012 and 11178002.

Open Access This article is distributed under the terms of the Creative Commons Attribution License which permits any use, distribution, and reproduction in any medium, provided the original author(s) and the source are credited.

Funded by $\mathrm{SCOAP}^{3}$ / License Version CC BY 4.0.

\section{References}

1. P. Jaranowski, G. Schäfer, Phys. Rev. D 55, 4712 (1997)

2. N.E. Pati, C.M. Will, Phys. Rev. D 65, 104008 (2002)

3. T. Damour, P. Jaranowski, G. Schäfer, Phys. Rev. D 77, 064032 (2008)

4. T. Damour, P. Jaranowski, G. Schäfer, Phys. Rev. D 78, 024009 (2008)

5. J. Hartung, J. Steinhoff, Ann. Phys. 523, 919 (2011)

6. A. Nagar, Phys. Rev. D 84, 084028 (2011)

7. S. Hergt, G. Schäfer, Phys. Rev. D 78, 124004 (2008)

8. J. Steinhoff, S. Hergt, G. Schäfer, Phys. Rev. D 77, 081501 (2008)

9. M. Levi, Phys. Rev. D 85, 064043 (2012)

10. J. Levin, Phys. Rev. Lett. 84, 3515 (2000)

11. J.D. Schnittman, F.A. Rasio, Phys. Rev. Lett. 87, 121101 (2001)

12. N.J. Cornish, J. Levin, Phys. Rev. Lett. 89, 179001 (2002)

13. N.J. Cornish, J. Levin, Phys. Rev. D 68, 024004 (2003)

14. X. Wu, Y. Xie, Phys. Rev. D 76, 124004 (2007)

15. J. Levin, Phys. Rev. D 67, 044013 (2003)

16. C. Königsdörffer, A. Gopakumar, Phys. Rev. D 71, 024039 (2006)

17. A. Gopakumar, C. Königsdörffer, Phys. Rev. D 72, 121501(R) (2005)

18. J. Levin, Phys. Rev. D 74, 124027 (2006)

19. X. Wu, Y. Xie, Phys. Rev. D 81, 084045 (2010)

20. X. Wu, S.Y. Zhong, Gen. Relativ. Gravit. 43, 2185 (2011)

21. L. Mei, M. Ju, X. Wu, S. Liu, Mon. Not. R. Astron. Soc. 435, 2246 (2013)

22. Y. Wang, X. Wu, Class. Quantum Gravity 28, 025010 (2011)

23. M.D. Hartl, A. Buonanno, Phys. Rev. D 71, 024027 (2005)

24. X. Wu, Y. Xie, Phys. Rev. D 77, 103012 (2008)

25. A. Buonanno, Y. Chen, T. Damour, Phys. Rev. D 76, 104005 (2006)

26. L. Mei, X. Wu, F. Liu, Eur. Phys. J. C 73, 2413 (2013)

27. G. Tancredi, A. Sánchez, F. Roig, Astorn. J. 121, 1171 (2001)

28. X. Wu, T.Y. Huang, Phys. Lett. A 313, 77 (2003)

29. C. Froeschlé, E. Lega, Celest. Mech. Dyn. Astron. 78, 167 (2000)

30. X. Wu, T.Y. Huang, H. Zhang, Phys. Rev. D 74, 083001 (2006)

31. P.C. Peters, Phys. Rev. B 136, 1224 (1964) 